\title{
In vitro gastric cancer cell imaging using near-infrared quantum dot-conjugated CC49
}

\author{
YUN-PENG ZHANG $^{1}$, PENG SUN ${ }^{1}$, XU-RUI ZHANG ${ }^{2}$ and WU-LI YANG ${ }^{2}$ \\ ${ }^{1}$ Department of General Surgery, Huashan Hospital; ${ }^{2}$ Laboratory of Advanced Materials, \\ Fudan University, Shanghai 200040, P.R. China
}

Received May 24, 2012; Accepted August 8, 2012

DOI: $10.3892 / \mathrm{ol} .2012 .870$

\begin{abstract}
In this experiment, we developed a bioprobe label for immunofluorescence using gastric tumor-specific quantum dots (QDs) to detect gastric tumor cells in vitro. The fluorescent probe, which is capable of specifically labeling gastric tumor cells, was constructed by taking advantage of the unique and superior properties of QDs. We grafted primary QDs onto the tumor-associated glycoprotein 72 (TAG-72) monoclonal antibody CC49 to produce CC49-QDs that specifically label tumor cells. Following a series of tests on the diameter and emission spectrum of CC49-QDs, they were employed in immunofluorescence analysis. Transmission electron microscopy and fluorescence spectrum analyses indicated that CC49-QDs had a $0.25 \mathrm{~nm}$ higher average diameter than the primary QDs, and that the grafted CC49 had no difference in optical properties compared to the primary QDs. In cell imaging, the cells labeled with CC49-QDs generated brighter fluorescence compared with the cells of the primary QD group. The results of immunofluorescence analysis demonstrated that antibody grafting reinforced the specific binding of QDs to tumor cells. This probe may also be further applied to live gastric cancer animal models to track lymphatic metastasis. In addition, it may potentially offer theoretical support for lymphadenectomy in the treatment of gastric cancer.
\end{abstract}

\section{Introduction}

Gastric cancer remains a health-threatening disease. Its incidence ranks fourth among all cancers, with up to 80 new cases per 100,000 people every year in Asian countries such as Japan, South Korea, North Korea and China. Gastric cancer is the second most frequent cause of cancer-related mortality following lung cancer, and its mortality rate has reached $85 \%$ (1). Currently, surgery is the most common treatment

Correspondence to: Professor Peng Sun, Department of General Surgery, Huashan Hospital, Fudan University, 12 Wulumuqi Middle Road, Shanghai 200040, P.R. China

E-mail: sunpn@msn.com

Key words: CC49, TAG-72, quantum dots, near-infrared fluorescence, cell imaging for gastric cancer; however, controversy remains regarding the preferred method of lymph node clearance as neither D1 lymphadenectomy proposed by Western researchers nor D2 lymphadenectomy proposed by Japanese researchers can be achieved with high specificity (2). The tracking and imaging of gastric tumor cells hinders the development of treatments for gastric cancer.

In tumor imaging, the ideal method should be sensitive, accurate, rapid, noninvasive, nonradioactive and potentially useful in a laparoscopic setting. Previous sentinel lymph node (SLN) mapping has usually involved preoperative injection of a radioactive colloid tracer such as $99 \mathrm{mTc}$ sulfur colloid followed by an intraoperative injection of a visible blue dye such as isosulfan blue. However, these staining materials have deficits in imaging such as poor tissue contrast and difficult detection in deeper anatomical regions. There is also a high false-positive rate in lymphatic mapping using the blue dye. As with radioactive isotopes, the high radioactivity of the primary injection site may interfere with intraoperative in vivo detection of nearby nodes $(3,4)$. The emerging nanocrystal fluorescence material, namely semiconductor quantum dots (QDs) or semiconductor nanocrystals, are not only free from such problems, but also have unique optical properties. Under proper light excitation, semiconductor QDs of different sizes and materials emit a narrow and tunable spectrum $(5,6)$. Compared with organic dyes such as rhodamine, semiconductor QDs are 20 times brighter, 100 times more stable against photobleaching and are $1 / 3$ wider in spectral bandwidth. Moreover, this nanocrystal fluorescence material is water-soluble and biocompatible $(7,8)$. In addition, the surface area-to-volume ratio of semiconductor QDs is larger and may therefore be grafted onto the surface of certain specific biomolecules, such as peptides, polyethylene, ligands and antibodies $(9,10)$. However, QDs have their shortcomings. Primary QDs, driven by internal circulation, fail to bind specifically to target proteins and remain in place. On this basis, we grafted primary QDs onto the monoclonal antibody CC49 for the purpose of obtaining CC49-QDs that not only bind specifically to tumor cells but also maintain the desired optical properties. These QDs therefore work as bioprobes in immunofluorescence imaging (11).

In this study, tumor-associated glycoprotein TAG-72 was adopted, with a macromolecular weight of approximately 220 to $400 \mathrm{kDa}(12)$, as it is expressed on the surface of or in tumor cells in a variety of cancers, such as colonic adenocarcinoma, 
invasive ductal carcinoma of the breast, non-small cell lung carcinoma, epithelial ovarian carcinoma, and pancreatic and gastric cancers, while it cannot be expressed in normal tissues and cells (13). The expression rate of TAG-72 in gastric cancers has been revealed to be as high as $75 \%$ (14). CC49 was adopted as the antibody. The first generation antibody B72.3 and the second generation $\mathrm{CC}$ antibodies are both commonly used to react with TAG-72. The CC series, comprised of 28 antibodies, was purified from the first generation antibody and has been shown to be capable of reacting with TAG-72 via both radioimmunoassay and immunohistochemical analyses. According to the reactive results of $\mathrm{CC}$ antibodies in a directbinding radioimmunoassay to a range of human carcinomas, western blotting, live cell surface binding assays, five liquid competition radioimmunoassays and $\mathrm{Ka}$ measurements, nine CC antibodies (CC11 CC15, CC29, CC30, CC40, CC46, CC49, CC83 and CC92) were selected for further analyses $(15,16)$. Thus, CC49 was selected, as it has a much higher Ka than B72.3 (16×10 $10^{9}$ vs. $\left.2.5 \times 10^{9} / \mathrm{mol}\right)$, to bind to primary QDs to synthesize CC49-QDs. These CC49-QDs are able to specifically bind to gastric tumor cell antigens (17).

It has been found that following injection into living animals, the localization of QDs may be monitored via fluorescence imaging; i.e., QDs can be used in vivo $(18,19)$. Therefore, CC49-QDs were synthesized by binding primary QDs to CC49 antibodies in the hope that CC49-QDs could hold on to the target protein via an antibody-antigen reaction and then be observed via fluorescence imaging. However, the synthesis of CC49-QDs may lead to size changes that directly affect optical properties, which may further exert an influence on their feasibility in animal experiments and clinical practice. Hence, in this investigation, primary QDs and CC49-QDs were analyzed to assess potential size changes and subsequent wavelength changes caused by the size changes, using spectrum analysis. Thereafter, both primary QDs and CC49-QDs were used to label gastric tumor cells with immunofluorescence. Meanwhile, a blank control group and a positive control group with fluorescein isothiocyanate (FITC)-labeled secondary antibodies were also determined.

\section{Materials and methods}

Materials and apparatus. Cadmium chloride $\left(\mathrm{CdCl}_{2}\right)$, 3-mercaptopropionic acid (MPA) and sodium borohydride $\left(\mathrm{NaBH}_{4}\right)$ were purchased from Acros Organics (Geel, Belgium). Tellurium powder was purchased from Sigma Aldrich (St. Louis, MO, USA). N-(3-dimethylaminopropyl)-N-ethylcarbodiimide hydrochloride (EDC) and N-hydroxysuccinimide (NHS) were supplied by Shanghai Medpep Co., Ltd, (Shanghai, China). The gastric cancer cell line MGC80-3 was supplied by the Shanghai Institute for Biological Sciences, Chinese Academy of Sciences. Certified fetal bovine serum (US) and bovine serum were supplied by Gibco (Grand Island, NY, USA). RPMI1640 and enhanced chemiluminescent (ECL) substrate were supplied by Hyclone (South Logan, UT, USA). Cell lysis buffer, phenylmethanesulfonyl fluoride (PMSF) and 4',6-diamidino2-phenylindole (DAPI) were supplied by Beyotime (Shanghai, China). The Enhanced BCA Protein Assay kit was supplied by BioTek (Highland Park, MI, USA). Goat-anti-mouse IgG $(\mathrm{H}+\mathrm{L})$ was supplied by Jackson Immunoresearch (West Grove,
PA, USA). Beta-actin (ACTB) antibody RabMAb was supplied by Epitomics (Burlingame, CA, USA). Polyvinylidene fluoride (PVDF) membranes $(0.45 \mu \mathrm{m})$ were supplied by Millipore (Billerica, MA, USA). CC49 monoclonal antibody and secondary antibody were purchased from Santa Cruz Biotechnology Inc. (Santa Cruz, CA, USA). A transmission electron microscope (FEI Tecnai F20) purchased from FEI (Lausanne, Switzerland) and a Fluorescence Lifetime and Steady State Spectroscopy (FLS920) apparatus purchased from Edinburgh Instruments (Livingston, UK) were the main imaging devices used in this experiment. SuperSignal West Femto (FUJIFILM LAS-3000) was purchased from Thermo Fisher Scientific (Waltham, MA, USA) and a fluorescence microscope (NIKON 80i) was purchased from Nikon (Tokyo, Japan).

Synthesis of cadmium telluride (CdTe) QDs. The synthesis of CdTe QDs has been described in detail in other studies $(20,21)$. The first step was to prepare a sodium hydrogen telluride (NaHTe) solution. $\mathrm{NaBH}_{4}(100 \mathrm{mg})$ was dissolved into $20 \mathrm{ml}$ distilled water. In an ice-water bath, the solution was deoxidized under steady nitrogen flow for $30 \mathrm{~min}$. Tellurium powder (127 mg; $1 \mathrm{mmol}$ ) was quickly added into the solution. With continuous steady nitrogen flow and vigorous stirring, a clear purple NaHTe solution was successfully produced. The second step was to prepare a $\mathrm{CdCl}_{2}$-MPA solution. $\mathrm{CdCl}_{2}$ (366.6 mg; $2 \mathrm{mmol}$ ) was injected into $100 \mathrm{ml}$ distilled water to produce a solution with a concentration of $20 \mathrm{mmol}$. MPA (382.1 mg; $3.6 \mathrm{mmol}$ ) was added to ensure a molar ratio of $\mathrm{Cd}^{2+}$ to MPA of $1: 1.8$. The $\mathrm{pH}$ level was adjusted to 9.0 by adding $2 \mathrm{~mol} / \mathrm{l} \mathrm{NaOH}$ solution dropwise. The final step was the preparation of CdTe. NaHTe solution $(1 \mathrm{ml})$ and $\mathrm{CdCl}_{2}$-MPA $(20 \mathrm{ml})$ were mixed and stirred rapidly. The mixture precursor solution $(9 \mathrm{ml})$ was transferred into a teflon-lined stainless steel autoclave which was subsequently placed in a drying oven at $185^{\circ} \mathrm{C}$ for a set amount of time. The precipitation products were washed three times by ethanol and then placed into a vacuum drying oven at $40^{\circ} \mathrm{C}$. Primary QDs were thus obtained.

Synthesis of CC49-QDs. The preparation of CC49-QDs involved EDC and NHS, which functioned as cross-linkers, TAG-72 monoclonal antibody CC49 and near-infrared CdTe QDs, and was performed by mixing $13.5 \mu \mathrm{l}$ EDC (0.1 mM ), 13.5 $\mu \mathrm{l}$ NHS and $50 \mu \mathrm{l}$ QD solution. After shaking for $0.5 \mathrm{~h}$ at room temperature, $594 \mu 1 \mathrm{CC} 49$ monoclonal antibodies were added, resulting in a 1:4 ratio of CdTe to antibody. Another $2 \mathrm{~h}$ was needed for reaction at room temperature prior to centrifugation, which was performed four times using a $100 \mathrm{~K}$ ultra filter at 5,000 rpm for $15 \mathrm{~min}$. Each time, the lower strata liquids were discarded, and the supernatant products were diluted by $200 \mu \mathrm{l}$ PBS prior to subsequent centrifugation. The final product was diluted by PBS ( $\mathrm{pH} 7.4)$ and stored in a refrigerator at $4^{\circ} \mathrm{C}(22)$.

$Q D$ and CC49-QD electron microscopy and spectrum analysis. The prepared primary QDs and CC49-QDs were separately diluted in deionized water and several drops were applied to two pieces of carbon film supported by a copper mesh. When the water volatilized, they were placed under an 


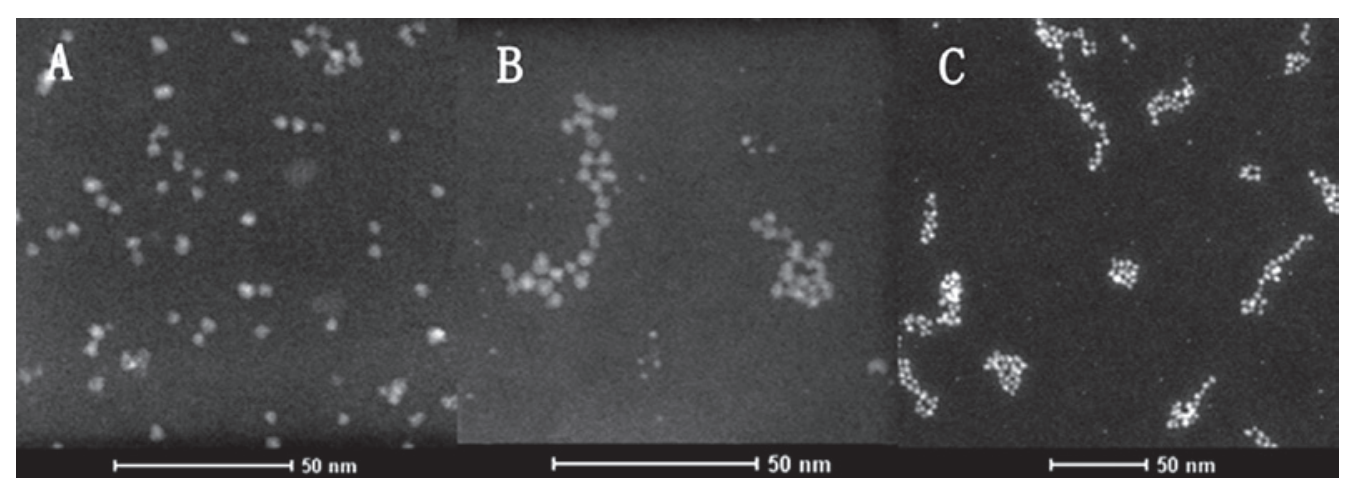

Figure 1. QD and CC49-QD electron microscopy. (A) Primary QD electron microscopy results; (B and C) Antibody-bound targeted QD electron microscopy results.

electron microscope which was adjusted to $200 \mathrm{~V}$ under the stem mode for observation. Images were then obtained.

Diluted QDs and CC49-QDs were placed under a spectrofluorimeter with a $450 \mathrm{~nm}$ excitation wavelength and a $1 \mathrm{~mm}$ slit. The curves of the spectra were drawn by recording the intensities of each nanometer of emission light between 550 and $800 \mathrm{~nm}$.

Antigen expression analysis. For protein extraction and quantification, cells were cultured in $10 \%$ fetal bovine serum, which was diluted by modified RPMI-1640 in a $\mathrm{CO}_{2}$ incubator. Approximately $10^{6}$ cells were washed twice in precooled PBS, and $395 \mu 1$ cell lysis buffer and $5 \mu 1$ PMSF were added. The cells were lysed on ice for $30 \mathrm{~min}$, scraped using a cell scraper and transferred into a $1.5 \mathrm{ml} \mathrm{EP}$ tube to be stored at $4^{\circ} \mathrm{C}$ for another $30 \mathrm{~min}$. They were subsequently centrifuged at 12,000 rpm for $5 \mathrm{~min}$ at $4^{\circ} \mathrm{C}$ and the supernatant was quantified using a BCA Protein Assay kit. The supernatant (40 $\mu \mathrm{l})$ was mixed with SDS sample buffer and packed into a $0.2 \mathrm{ml}$ EP tube. This was used in the western blot analysis following heating.

For the western blot analysis, samples containing approximately $80 \mu \mathrm{g}$ protein were mixed with SDS sample buffer and set in a $100^{\circ} \mathrm{C}$ heating block for $5 \mathrm{~min}$ prior to separation on a board with a $6 \%$ spacer gel and $8 \%$ separation gel under $120 \mathrm{~V}$. The protein was then transferred onto a PVDF membrane along with a spectra multicolor broad range protein ladder. Following the transfer, the PVDF membrane was blocked with 5\% non-fat dry milk in TBS/0.1\% Tween-20 (TBST) for $2 \mathrm{~h}$ on the shaker and then incubated with the monoclonal antibody CC49 at a dilution of 1:500. Given that TAG-72 was our target protein, whose molecular weight is between 220 and $240 \mathrm{kDa}$, beta-actin was adopted at approximately $40 \mathrm{kDa}$ as a housekeeping protein and it was detected with the beta-actin antibody RabMAb diluted to 1:1,000. The relative intensity of the beta-actin protein bands for the cell line was used to evaluate the amount of the sample protein that was loaded. Following an overnight incubation at $4^{\circ} \mathrm{C}$ the PVDF membrane was washed 3 times, each for $5 \mathrm{~min}$ in TBST at room temperature. Thereafter, the secondary antibody, HRP-linked anti-mouse IgG $(\mathrm{H}+\mathrm{L})$, diluted at 1:2,000, was added and the membrane was incubated for $1 \mathrm{~h}$ at room temperature and washed again 3 times for 5 min with TBST. Following incubation of the membrane for $1 \mathrm{~min}$, the protein was visualized with ECL substrate and subsequently exposed for $30 \mathrm{sec}(12)$.

Immunofluorescence. Cells were distributed into four 6-well chamber slides and labeled as group 1,2, 3 or 4. Group 1, the blank group, formed the negative control group. Group 2 was set as the CC49-QD group and Group 3 was set as the primary QD group. Group 4, the fluorescent secondary antibody group, was the positive control group. All four slides were incubated in a $\mathrm{CO}_{2}$ incubator until the cells covered the bottom of the wells. The culture solution was then removed and the cells were washed three times with PBS, each for $5 \mathrm{~min}$. Another three washes for 5 min each with PBS were needed after the cells were incubated in $4 \%$ paraformaldehyde for $15 \mathrm{~min}$. The cells were then blocked in $10 \%$ bovine serum for $30 \mathrm{~min}$ and subjected to three more washes. Subsequently, $1 \mathrm{ml} \mathrm{PBS,} 1 \mathrm{ml}$ CC49-QDs, $1 \mathrm{ml}$ primary QDs and $1 \mathrm{ml}$ CC49 monoclonal antibody, diluted to 1:500 with PBS, were added into the wells of groups 1-4, respectively. Following incubation for $2 \mathrm{~h}$ in an incubator at $37^{\circ} \mathrm{C}$, groups 1-3 were subjected to nuclear staining using DAPI for $5 \mathrm{~min}$, and then washed three times with PBS (each for $3 \mathrm{~min}$ ) before being observed under a fluorescent microscope. Group 4, prior to observation, underwent three PBS washes (each for $3 \mathrm{~min}$ ), then $1 \mathrm{ml}$ fluorescent secondary antibody was added and diluted to $1: 1,000$ with PBS. Group 4 cells were subsequently incubated at $37^{\circ} \mathrm{C}$ for $1 \mathrm{~h}$, stained using DAPI and finally washed again three times each for $5 \mathrm{~min}$. Thus, immunofluorescent images of the four groups were obtained (23).

\section{Results}

$Q D$ and CC49-QD electron microscopy and spectrum analysis. Transmission electron microscopy revealed that the diameters of QDs were approximately 2.24 to $4.91 \mathrm{~nm}$, averaging $3.47 \mathrm{~nm}$ (Fig. 1A). The diameters of CC49-QDs were approximately 3.30 to $5.65 \mathrm{~nm}$, averaging $3.72 \mathrm{~nm}$ (Fig. 1B), with a $0.25 \mathrm{~nm}$ higher average than that of QDs. Fig. 1B and C shows how certain CC49-QDs assembled together, resulting in the formation of QD clusters of different diameters from 7.69 to $55.77 \mathrm{~nm}$, averaging $23.76 \mathrm{~nm}$.

With the ordinate denoting light intensity and the abscissa denoting wavelength, the spectrum curves for QDs and 


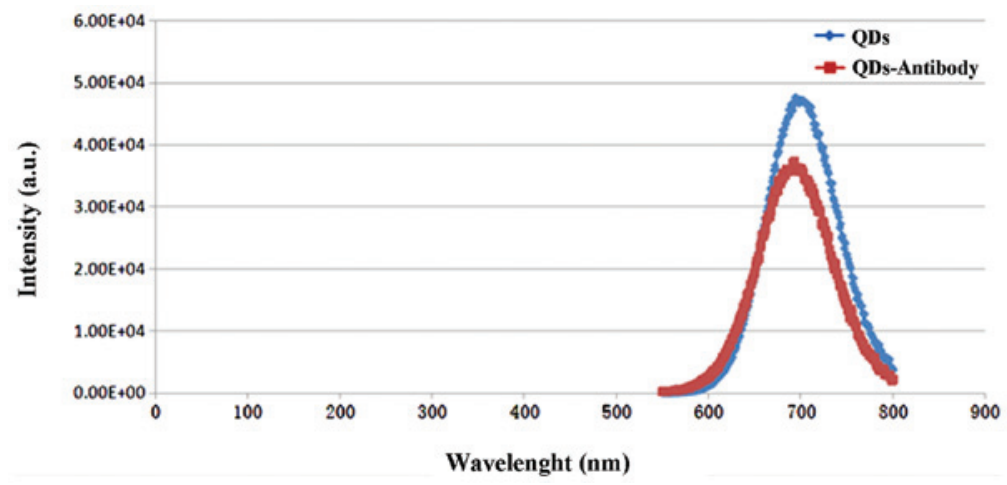

Figure 2. QDs and CC49-QD spectrum analysis. Blue denotes the primary QD spectrum analysis curve and red denotes the antibody-bound targeted QD spectrum analysis curve. QDs, quantum dots.

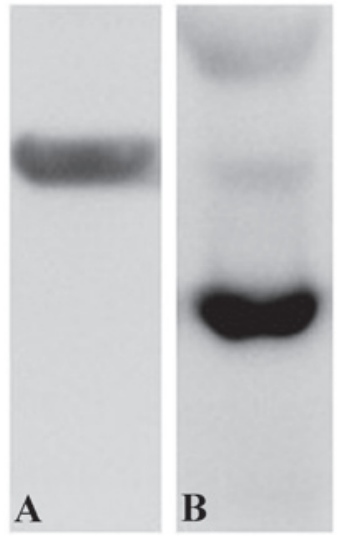

Figure 3. Western blot analysis. (A) Western blot analysis of TAG-72 in MGC80-3. (B) shows the beta-actin results, which served as an internal loading control.
CC49-QDs were drawn. As shown in Fig. 2, the emission light wavelengths of primary QDs were between 620 and $780 \mathrm{~nm}$, and the peak appeared at approximately $680 \mathrm{~nm}$. The wavelengths of the CC49-QD emission light were between 600 and $800 \mathrm{~nm}$, and the peak appeared at approximately $710 \mathrm{~nm}$. Although the light dimmed slightly following grafting, it was still significantly stronger than that of organic dyes.

Western blotting. In the experiment, the protein contents of $1,2,4$ and $8 \mu 1$ protein solutions were measured to be 1.849 , $3.702,7.214$ and $15.515 \mu \mathrm{g} / \mu \mathrm{l}$, respectively. When the volume of the sample exceeded $12 \mu 1$, the content was beyond the capability of the measuring device. Consequently, we conjectured, based on the above measurement of the protein content of the $40-\mu 1$ sample in the western blot, that is was approximately

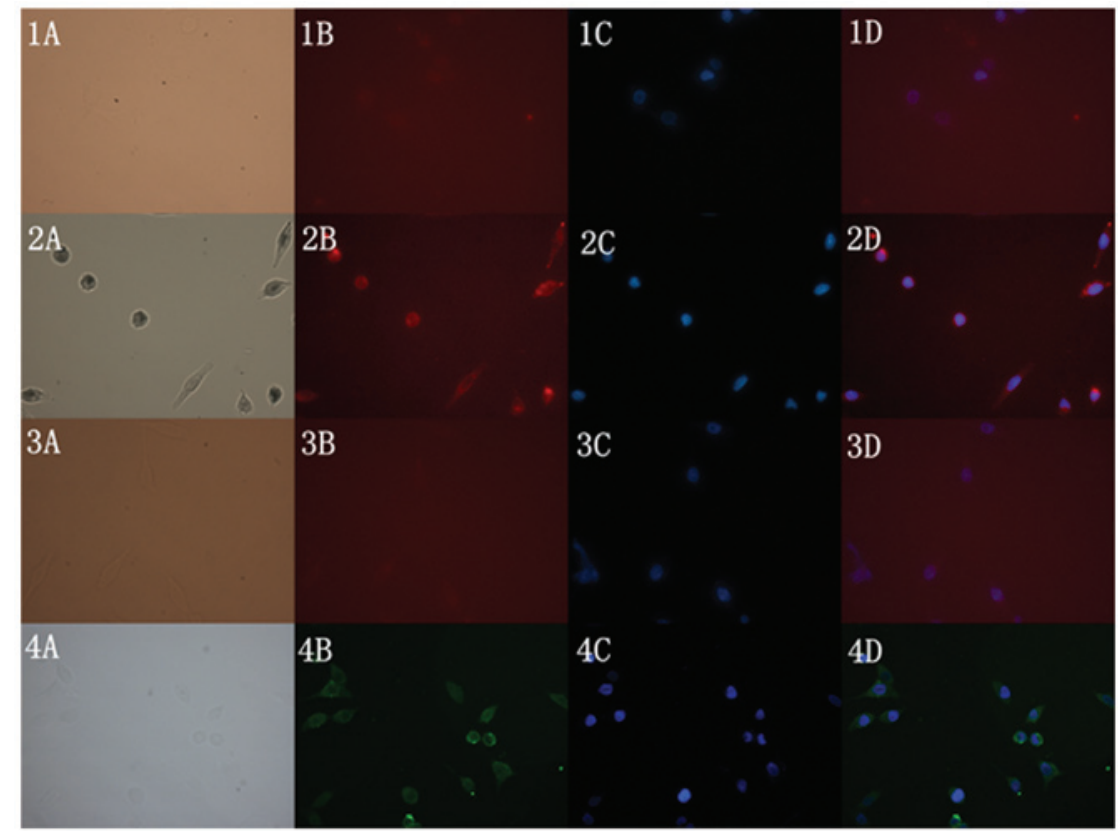

Figure 4. Gastric tumor cell fluorescence images representing (1A/1B/1C/1D) the blank control group; $(2 \mathrm{~A} / 2 \mathrm{~B} / 2 \mathrm{C} / 2 \mathrm{D})$ the experimental targeted $\mathrm{QD}$ group; $(3 \mathrm{~A} / 3 \mathrm{~B} / 3 \mathrm{C} / 3 \mathrm{D})$ the negative control group labeled by primary $\mathrm{QDs}$; and $(4 \mathrm{~A} / 4 \mathrm{~B} / 4 \mathrm{C} / 4 \mathrm{D})$ the positive control group labeled by secondary antibodies. $1 \mathrm{~A}, 2 \mathrm{~A}, 3 \mathrm{~A}$ and $4 \mathrm{~A}$ are optical microscopy images. 1B, 2B, and 3B are cell fluorescence images observed under the near-infrared band mode and $4 \mathrm{~B}$ is a cell fluorescence image observed under FITC mode. The C images represent the DAPI-stained nuclei under ultraviolet light mode and the D images are the merging of B and C images. QD, quantum dot. 
$80 \mu \mathrm{g}$. As a result, two protein images were obtained; one was the dark-colored beta-actin image (Fig. 3B) and the other was the light-colored TAG-72 image (Fig. 3A).

Immunofluorescent imaging. The images of the four groups were all observed under an optical microscope and fluorescence microscope. The blank group had an unclear image under the optical microscope [Fig. 4(1A)] and the fluorescence microscope [Fig. 4(1B], while the nuclei stained by DAPI were observed clearly under the ultraviolet excitation light [Fig. 4(1C)]. For the CC49-QD group, a clear image was observed both under the optical microscope [Fig. 4(2A)] and the fluorescence microscope [Fig. 4(2B)]. The nuclei stained by DAPI were also observed clearly under the ultraviolet excitation light [Fig. 4(2C)]. From Fig. 4(2D), which was formed by merging Fig. 4(2B) and (2C), we found that the highlighted cell fluorescence image overlapped with the nucleus image perfectly. In contrast, for the primary QD group, it was hard to distinguish the cell image under the optical microscope [Fig. 4(3A)] and the fluorescence microscope [Fig. 4(3B)]; however, the DAPI-stained nuclei were observed [Fig. 4(3C)]. In the positive control group, a vague cell image was visible under the optical microscope. Since it was labeled by a fluorescent secondary antibody with a FITC wavelength, a highlighted and clear cell fluorescent image could be visualized under the FITC stem mode [Fig. 4(4B)]. The DAPI-stained nucleus image was also observed clearly [Fig. 4(4C)]. Fig. 4(4D) was the result of merging Fig. 4(4B) and $(4 \mathrm{C})$, denoting the cell fluorescence image and the nucleus overlap.

\section{Discussion}

For non-invasive imaging, near-infrared QDs are better than traditional organic dyes in many ways and manifest great potential value in the clinical detection and treatment of cancer. Therefore, in this experiment, they were adopted to bind with CC49 monoclonal antibodies to form CC49-QDs. The synthesized CC49-QDs worked as a specific bioprobe and were employed in immunofluorescence imaging. In addition, CC49-QDs may also be further applied to animal models of gastric cancer for tracking tumor metastasis.

In this study, the near-infrared CdTe QDs were synthesized by a hydrothermal method $(23,24)$. There are two commonly used methods for QD preparation: organic metal synthesis and aqueous medium synthesis. The former, also named TOP-TOPO (trioctylphosphine oxide-trioctylphosphine) is widely used and has high quantum yields; however, it also has a high cost and demands strict external conditions. In addition, certain materials that are needed, such as dimethylcadmium, are highly toxic and harmful to health. Above all, the CC49-QDs prepared by this route are not able to dissolve into the internal environment due to low solubility unless subjected to complicated modification. All of these factors prevent wider use and extensive research using QDs. Hence, following the development of synthetic techniques and the ability to grow in yields, aqueous medium synthesis predominates due to its simplicity and reproducibility. Materials used in this method are of lower toxicity and do not demand strict external conditions, which lead to a significant cost reduction. In addition,
QDs produced by this method dissolve in the internal environment easily and may be stored for up to 2 years after drying. The bioprobe made in this way, bearing higher solubility and smaller size, promotes more effective in vivo fluorescence imaging (24).

With bioprobe production, near-infrared QDs occupy many superior optical properties compared to traditional organic dyes. They have a larger Stoke's shift (the wavelength difference between the excitation light and emission light), which helps avoid overlap of emission and excitation lights and thereby enhances the sensitivity of immunofluorescence (25). The Stoke's shift of organic fluorescence dyes is smaller and their emission wavelengths are usually between 450 and $550 \mathrm{~nm}$. Within this range, strong background autofluorescence is produced from endogenous chromophores such as collagens, porphyrins and flavins, therefore the label fluorescence may be overwhelmed. For this reason, its utilization in bioluminescence imaging is confined (26). In contrast, nearinfrared QDs surmount the organic fluorescence dyes in the above-mentioned aspects. Furthermore, within the wavelength range of the near-infrared spectrum (650-900 nm) (27), the absorbance by water and red blood cells is low, absorption and scattering of optical photons in tissues is low and the optical photon emission intensity is the strongest. All these factors are favorable for producing clearer fluorescence images (28). As mentioned above, a 450-nm excitation light was adopted in our study to excite the primary QDs and CC49-QDs. The measurement revealed that the emission light wavelengths of primary QDs were between 620 and $780 \mathrm{~nm}$ and the peak appeared at approximately $680 \mathrm{~nm}$. The wavelengths of the CC49-QD emission light were between 600 and $800 \mathrm{~nm}$ and the peak appeared at approximately $710 \mathrm{~nm}$. The Stoke's shift was approximately $200-300 \mathrm{~nm}$ and the emission light was near infrared. This result demonstrated that the Stoke's shift of our CC49-QDs was large enough and its wavelength allowed the autofluorescence to dominate. More sensitive real-time imaging of tissues and cells can be achieved; therefore, the use of CC49-QDs as bioprobes in living animals is of great practical value.

QDs have advantages as a promising bioprobe material; however, certain disadvantages have hindered its practical use. For example, primary QDs tend not to conduct specificity in binding to target proteins due to surface carboxylic acid and amines. Bulks may grow due to their non-specific binding to cellular membranes, proteins and extracellular matrix materials. In any case, it is the non-specificity that causes a high level of background fluorescence and false-positive results in imaging. Therefore, the surface of QDs needs modification for practical application, including direct ligand exchange reactions and indirect surface encapsulation. Materials used in surface coating are silica, lipid and amphiphatic molecular polymers $(29,30)$. In the present study, an MPA coating was used to minimize the non-specific absorption. We demonstrated that MPA markedly reduced exposed free carboxylic acids, prevented non-specific binding and guaranteed the stability of QDs in water solution. In this way, non-specific binding did not blur the imaging (31).

The optical properties of QDs are size-dependent; that is, when excited by proper light, QDs of different sizes emit narrow and tunable lights of different wavelengths. It follows 
that the size of QDs defines their optical properties and proceeds to impact their use in immunofluorescence imaging as bioprobes. The surface coating and CC49 monoclonal antibody may impact the QD size and optical properties, which seemingly countered our initial design. To avoid this, we first measured the diameters of primary QDs and CC49-QDs under an optical microscope. This revealed that the diameters of QDs were approximately 2.24 to $4.91 \mathrm{~nm}$, averaging $3.47 \mathrm{~nm}$ (Fig. 1A), while those of the CC49-QDs were approximately 3.30 to $5.65 \mathrm{~nm}$, averaging $3.72 \mathrm{~nm}$ (Fig. 1B). The difference was not significant and was only approximately $0.25 \mathrm{~nm}$; however, CC49-QDs gathered together and formed 7.69 to 55.77-nm-sized QD bulks in solution, with an average size of $23.76 \mathrm{~nm}$. This change was significant in relation to primary QDs. Consequently, a spectrum analysis was needed to judge its availability. Fig. 2 shows the analysis results. The emission light wavelengths of modified QDs were between 620 and $780 \mathrm{~nm}$, while those of CC49-QDs emission lights were between 600 and $800 \mathrm{~nm}$. With a 480-nm excitation light, their emission spectrum regions were approximately the same. Thus, it was justified to use CC49-QDs for tumor cell imaging since the modification did not change the optical properties.

The most superior property of the prepared CC49-QDs, however, lay in the binding specificity of the CC49 monoclonal antibody to gastric tumor cell antigen TAG-72. Due to this specificity, we achieved fluorescence labeling of gastric tumor cells using CC49-QDs. In other words, the presence of TAG-72 served as the prerequisite for immunofluorescence imaging in our study. To justify the feasibility of immunofluorescence imaging, western blotting was adopted to determine the characterization of MGC80-3 gastric tumor cell lines. It verified the existence of TAG-72 in gastric tumor cell lines.

As for the results of immunofluorescence imaging, only vague cell images were observed in the primary QD group as in the negative control group. Compared with the images in the positive control group, the intensity was similar. By contrast, in the CC49-QD group, the cell boundaries were clear and the intensity was even stronger than in the positive control group. This result indicated that there were many more QDs binding to gastric tumor cells in the target QD group than in the primary QD group. To confirm the binding, DAPI was used to stain the nucleus in order to locate cells. In this way, a perfect overlap could be obtained, and it was found that the stained nuclei were lying in the center of the cells, surrounded by the fluorescence boundaries. We thereby confirmed specific binding. The study demonstrated the specific binding of CC49-QD probes to gastric tumor cells, which facilitated immunofluorescence labeling. In addition, the detection of their optical properties demonstrated their advantages as a type of bioprobe. These results provide experimental support for tracking gastric tumor cell metastasis in gastric tumor animal models.

\section{Acknowledgements}

This study was supported by the National Nature Science Foundation of China (No. 20874015) and the Science and Technology Commission Nano Special Fund of the Shanghai Municipality (No. 1052nm03802).

\section{References}

1. Krejs GJ: Gastric cancer: epidemiology and risk factors. Dig Dis 28: 600-603, 2010

2. Japanese Gastric Cancer Association: Japanese gastric cancer treatment guidelines. Gastric Cancer 14: 113-123, 2011.

3. Soltesz EG, Kim S, Kim SW, Laurence RG, Grand AM, Parungo CP, Cohn LH, Bawendi MG and Frangioni JV: Sentinel lymph node mapping of the gastrointestinal tract by using invisible light. Ann Surg Oncol 13: 386-396, 2006.

4. Ohnishi S, Lomnes SJ, Laurence RG, Gogbashian A, Mariani G and Frangioni JV: Organic alternatives to quantum dots for intraoperative near-infrared fluorescent sentinel lymph node mapping. Mol Imaging 4: 172-181, 2005.

5. Michalet X, Pinaud FF, Bentolila LA, Tsay JM, Doose S, Li JJ, Sundaresan G, Wu AM, Gambhir SS and Weiss S: Quantum dots for live cells, in vivo imaging, and diagnostics. Science 307: 538-544, 2005.

6. Alivisatos AP, Gu W and Larabell C: Quantum dots as cellular probes. Annu Rev Biomed Eng 7: 55-76, 2005.

7. Chan WC and Nie S: Quantum dot bioconjugates for ultrasensitive nonisotopic detection. Science 281: 2016-2018, 1998.

8. Åkerman ME, Chan WC, Laakkonen P, Bhatia SN and Ruoslahti E: Nanocrystal targeting in vivo. PNAS 99: 12617-12621, 2002.

9. Gao X, Yang L, Petros JA, Marshall FF, Simons JW and Nie S: In vivo molecular and cellular imaging with quantum dots. Curr Opin Biotechnol 16: 63-72, 2005.

10. Li ZG, Yang K, Cao YA, Zheng G, Sun DP, Zhao C and Yang J: In vivo study of the effects of peptide-conjugated near-infrared fluorescent quantum dots on the tumorigenic and lymphatic metastatic capacities of squamous cell carcinoma cell line Tca8113 and U14. Int J Mol Sci 11: 1413-1422, 2010.

11. Yang K, Zhang FJ, Tang H, Zhao C, Cao YA, Lv XQ, Chen D and Li YD: In vivo imaging of oral squamous cell carcinoma by EGFR monoclonal antibody conjugated near-infrared quantum dots in mice. Int J Nanomedicine 6: 1739-1745, 2011.

12. Chen L, Wang Y, Liu X, Dou S, Liu G, Hnatowich DJ and Rusckowski M: A new TAG-72 cancer marker peptide identified by phage display. Cancer Lett 272: 122-132, 2008.

13. Paterson AJ, Schlom J, Sears HF, Bennett J and Colcher D: A radioimmunoassay for the detection of a human tumor associated glycoprotein (TAG-72) using monoclonal antibody B72.3. Int J Cancer 37: 659-666, 1986.

14. Thor A, Ohuchi N, Szpak CA, Johnston WW and Schlom J: Distribution of oncofetal antigen tumor-associated glycoprotein-72 defined by monoclonal antibody B72.3. Cancer Res 46: 3118-3124, 1986.

15. Johnson VG, Schlom J, Paterson AJ, Bennett J, Magnani JL and Colcher D: Analysis of a human tumor - associated glycoprotein (TAG-72) identified by monoclonal antibody B72.3. Cancer Res 46: 850-857, 1986.

16. Muraro R, Kuroki M, Wunderlich D, Poole DJ, Colcher D, Thor A, Greiner JW, Simpson JF, Molinolo A, Noguchi P and Schlom J: Generation and characterization of B72.3 second generation monoclonal antibodies reactive with the tumorassociated glycoprotein 72 antigen. Cancer Res 48: 4588-4596, 1988.

17. Sheer DG, Schlom J and Cooper HL: Purification and composition of the human tumor-associated glycoprotein (TAG-72) defined by monoclonal antibodies CC49 and B72.3. Cancer Res 48: 6811-6818, 1988.

18. Ballou B, Lagerholm BC, Ernst LA, Bruchez MP and Waggoner AS: Noninvasive imaging of quantum dots in mice. Bioconjugate Chem 15: 79-86, 2004.

19. Gao X, Cui Y, Levenson RM, Chung LW and Nie S: In vivo cancer targeting and imaging with semiconductor quantum dots. Nat Biotechnol 22: 969-976, 2004.

20. Guo J, Yang W and Wang C: Systematic study of the photoluminescence dependence of thiol-capped CdTe nanocrystals on the reaction conditions. J Phys Chem B 109: 17467-17473, 2005.

21. Zhang $\mathrm{H}$, Wang L, Xiong $\mathrm{H}$, $\mathrm{Hu} \mathrm{L}$, Yang $\mathrm{B}$ and $\mathrm{Li} \mathrm{W}$ : Hydrothermal synthesis for high-quality CdTe nanocrystals. Adv Mater 15: 1712-1715, 2003.

22. Hu M, Yan J, He Y, Lu H, Weng L, Song S, Fan C and Wang L: Ultrasensitive, multiplexed detection of cancer biomarkers directly in serum by using a quantum dot-based microfluidic protein chip. ACS Nano 4: 488-494, 2010. 
23. Zou P, Xu S, Povoski SP, Wang A, Johnson MA, Martin EW Jr, Subramaniam V, Xu R and Sun D: Near-infrared fluorescence labeled anti-TAG-72 monoclonal antibodies for tumor imaging in colorectal cancer xenograft mice. Mol Pharm 6: 428-440, 2009.

24. Gaponik N, Talapin DV, Rogach AL, Hoppe K, Shevchenko EV, Kornowski A, Eychmuller A and Weller H: Thiol-capping of CdTe nanocrystals: an alternative to organometallic synthetic routes. J Phys Chem B 106: 7177-7185, 2002.

25. Jaiswal JK and Simon SM: Potentials and pitfalls of fluorescent quantum dots for biological imaging. Trends In Cell Biol 14: 497-504, 2004

26. So MK, Xu C, Loening AM, Gambhir SS and Rao J: Selfilluminating quantum dot conjugates for in vivo imaging. Nat Biotechnol 24: 339-343, 2006.

27. Cheng Z, Wu Y, Xiong Z, Gambhir SS and Chen X: Near-infrared fluorescent RGD peptides for optical imaging of integrin $\alpha \mathrm{V} \beta 3$ expression in living mice. Bioconjugate Chem 16: 1433-1441, 2005.
28. Tanisaka H, Kizaka-Kondoh S, Makino A, Tanaka S, Hiraoka M and Kimura S: Near-infrared fluorescent labeled peptosome for application to cancer imaging. Bioconjugate Chem 19: 109-117, 2008.

29. Kairdolf BA, Mancini MC, Smith AM and Nie S: Minimizing nonspecific cellular binding of quantum dots with hydroxylderivatized surface coatings. Anal Chem 80: 3029-3034, 2008.

30. Bentzen EL, Tomlinson ID, Mason J, Gresch P, Warnement MR, Wright D, Sanders-Bush E, Blakely R and Rosenthal SJ: Surface modification to reduce nonspecific binding of quantum dots in live cell assays. Bioconjugate Chem 16: 1488-1494, 2005.

31. Gerion D, Pinaud F, Williams SC, Parak JW, Zanchet D, Weiss S and Alivisatos AP: Synthesis and properties of biocompatible water-soluble silica-coated $\mathrm{CdSe} / \mathrm{ZnS}$ semiconductor quantum dots. J Phys Chem B 105: 8861-8871, 2001. 